\title{
Management Dilemma of an Infertile Patient with More Than 20 Submucous Fibroids
}

\author{
Ahmed Abdelaziz, MD,,2 Salem Joseph, BS, ${ }^{1}$ Mohamed Ashraf, MD, ${ }^{1-3}$ and Mostafa I. Abuzeid, MD ${ }^{1-3}$
}

\begin{abstract}
Background: Submucous fibroids can lead to menorrhgia, infertility, recurrent pregnancy loss, and obstetric complications. The management of multiple submucous fibroids in patients who would like to preserve their reproductive function can be a challenge. The aim of this report is to discuss the management dilemma of a patient who presented with multiple (more than 20) submucous fibroids. Case: The patient was a 33-year-old Caucasian woman who had an initial complaint of menometrorrhagia and secondary infertility, which led to a diagnostic hysteroscopy. Multiple submucosal fibroids were noted; no other etiology for infertility was identified. She subsequently underwent hysteroscopic resection of several fibroids. Postoperative hyterosalpingogram demonstrated intrauterine scarring, unilateral tubal blockage, and several remaining fibroids. She did, however, conceive with intrauterine insemination. Unfortunately, this pregnancy ended in an early spontaneous abortion of twins. After being counseled on her options, including the possibility of needing a surrogate uterus, she elected to have an abdominal resection of the remaining fibroids. During this procedure, several steps were taken to restore the uterine cavity to a normal shape. After allowing her uterus to heal, she achieved pregnancy through in vitro fertilization. At 29 weeks of gestation, she had preterm premature rupture of membranes and, subsequently, had a cesarean section at 34 weeks of gestation. She had a viable 6 pound 1 ounce baby boy. At the time of her cesarean section, only one submucosal fibroid was identified. Conclusion: We present a unique case of multiple sub-mucous fibroids that failed hysteroscopic management and were subsequently treated successfully with hysterotomy, myomectomy, and uterine reconstructive surgery. Based on our experience with this case, we recommend hysterotomy and myomectomy for management of multiple sub-mucous fibroids from the outset.
\end{abstract} ( J GYNECOL SURG 31:274)

\section{Introduction}

$\mathbf{U}$ TERINE FIBROIDS ARE the most common benign gynecologic tumors. They are benign clonal tumors that arise from the smooth muscle cells of the uterus. They cause a variety of symptoms, including menometorrhagia, dysmenorrhea, pelvic pain, infertility, preterm birth, and compression of adjacent pelvic viscera. ${ }^{1}$ Although fibroids can present at any age, most women become symptomatic during their $30 \mathrm{~s}^{2}$

A number of theories have been suggested as to the cause and effect relationship of myomas on fertility. Pritts performed a review of the literature and found that fibroids may contribute to infertility via several possible mechanisms, including blockage of the tubal Ostia, abnormal vascularization, abnormal endometrial development, promotion of chronic intra-cavitary inflammation, and increasing uterine contractility. ${ }^{3}$ Some investigators suggested that the hyper estrogenic state causes anovulatory cycle $^{2}$; elongation and distortion of the glands may cause implantation failure, , $^{2,4}$ while others have suggested that myomas may cause dysfunctional uterine contractility, which may interfere with sperm migration, ovum transport. ${ }^{5}$

Studies have shown that submucosal fibroids are associated with an increased risk of spontaneous abortion, preterm delivery, malpresentation, pelvic outlet obstruction, postpartum hemorrhage, and puerperal sepsis. ${ }^{6}$ Myomectomy is usually performed in an effort to preserve fertility. Surgical options include a vaginal approach via hysteroscopic resection or an abdominal approach via laparotomy or laparoscopy. The utility of myomectomies for the treatment of infertility remains controversial if no other cause for infertility can be identified. It is logical that submucosal myomas, which

\footnotetext{
${ }^{1}$ Division of Reproductive Endocrinology and Infertility, Department of Obstetrics and Gynecology, Hurley Medical Center, Flint, MI. ${ }^{2}$ College of Human Medicine, Michigan State University, Flint, MI.

${ }^{3}$ IVF Michigan, Rochester Hills, MI.
} 


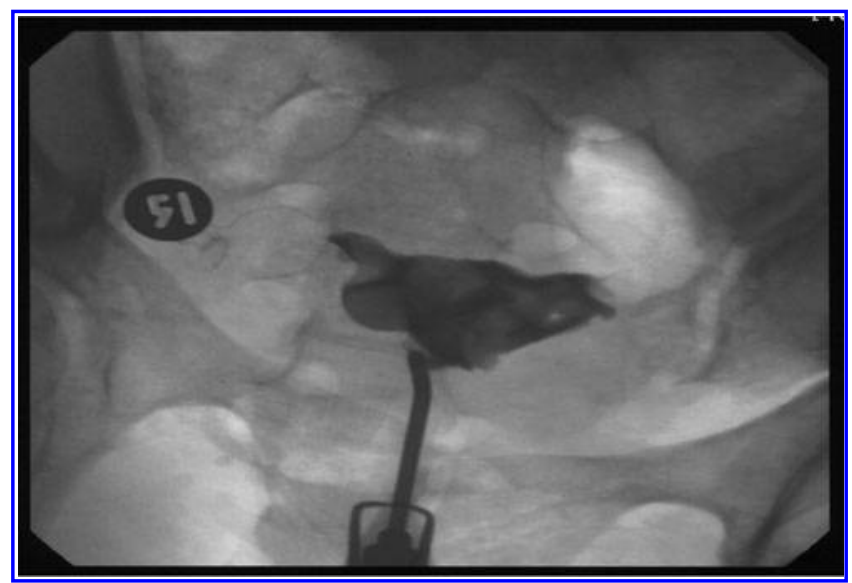

FIG. 1. Preoperative hyterosalpingogram showing several submucous fibroids.

obstruct or distort the uterine cavity, may affect implantation or be a source of recurrent miscarriages. If a submucosal fibroid is identified, a hysteroscopic resection is the best and most effective surgical approach. ${ }^{1}$ If the submucosal fibroid appears to be buried within the myometrium, and cannot be removed completely by hysteroscopy, extreme caution should be used and a conversion to an abdominal approach may be the safest option.

We are reporting an unusual case of a viable preterm pregnancy and cesarean delivery in a woman who underwent an incomplete hysteroscopic resection and subsequent abdominal myomectomy of $>20$ submucosal fibroids as treatment for her infertility.

\section{Case Report}

A 33-year-old white woman G1P0010 was referred to our office for secondary infertility after trying to conceive for 1 year. Her medical history was significant for uterine fibroids and a termination of pregnancy in the past. Her gynecologic history revealed a 28-day cycle with menometrorrhagia and occasional dyspareunia. She was hospitalized for excessive vaginal bleeding and low hemoglobin $(7.2 \mathrm{gm} / \mathrm{dL})$ for which she received 2 units of packed RBCs. She was placed on lupron depot for 3 months to attempt to improve her symptomatology. Subsequently, she underwent a diagnostic hysteroscopy by her gynecologist that revealed multiple submucosal fibroids. A hysterosalpingram showed several submucosal fibroids and a normal fill and spill of the fallopian tubes bilaterally (Fig. 1).

She elected to undergo a diagnostic laparoscopy with tubal perfusion and possibly two to three steps of hysteroscopic myomectomy. The potential risks, benefits, and complications, including intrauterine scar formation, were discussed in details and the patient understood. The operative findings showed a normal abdomen, normal healthy fimbriae, normal ovaries, and no evidence of endometriosis (Fig. 2). The endometrial cavity was obliterated with more than 20 submucosal myomas $\sim 0.5$ to $1 \mathrm{~cm}$ in diameter (Fig. 3). Partial hysteroscopic myomectomy was performed. A 10F Foley catheter was introduced into the endometrial cavity and insufflated with $4 \mathrm{~mL}$ of normal saline. The balloon was left in place for 7 days. The patient was placed on estrogen $1.25 \mathrm{mg}$ b.i.d. for 6 weeks followed by medroxyprogesterone daily for 10 days.

Three months after surgery, her postoperative hysterosalpingogram demonstrated irregularity along the left uterine wall and an intrauterine scar obliterated the right upper uterine segment (Fig. 4). Therefore, the right fallopian tube did not fill or spill. Functionally, she had what appeared similar to the left unicornuate uterus. The left fallopian tube had normal fill and spill.

After discussing several assisted reproductive options, she opted to proceed with clomiphene and monitoring for three cycles, but she did not conceive. This was followed by a successful gonadotropin ovulation induction followed by intrauterine insemination, and she became pregnant with twins. Unfortunately, this ended in a spontaneous miscarriage at 8 weeks of gestation, which required dilation and curettage.

After this setback, she was provided with further options, including assisted reproduction technology using a gestational carrier, but she declined. She elected to undergo a repeat myomectomy. This time, the approach would be via a laparotomy with bi-halving of the uterus to excise the submucosal fibroids and lysis of adhesions. She was informed of the potential risks, benefits, and complications, including scar tissue formation in the uterus that may prevent her from

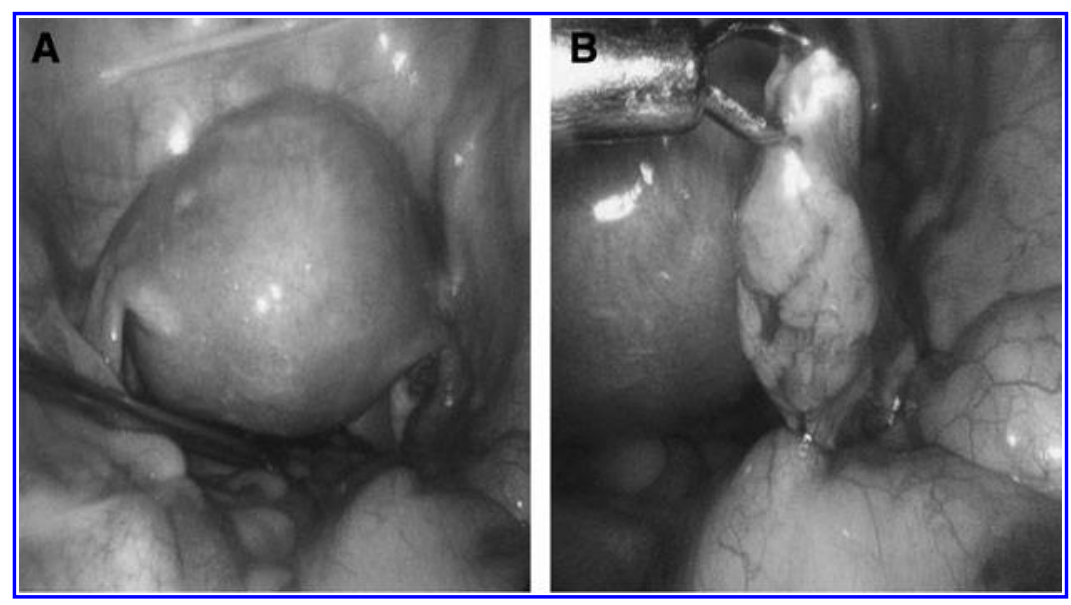

FIG. 2. (A, B) Laparoscopic picture showing enlarged uterus as a result of multiple submucous fibroids, no subserous fibroids, and normal healthy fimbria of the right fallopian tube. 


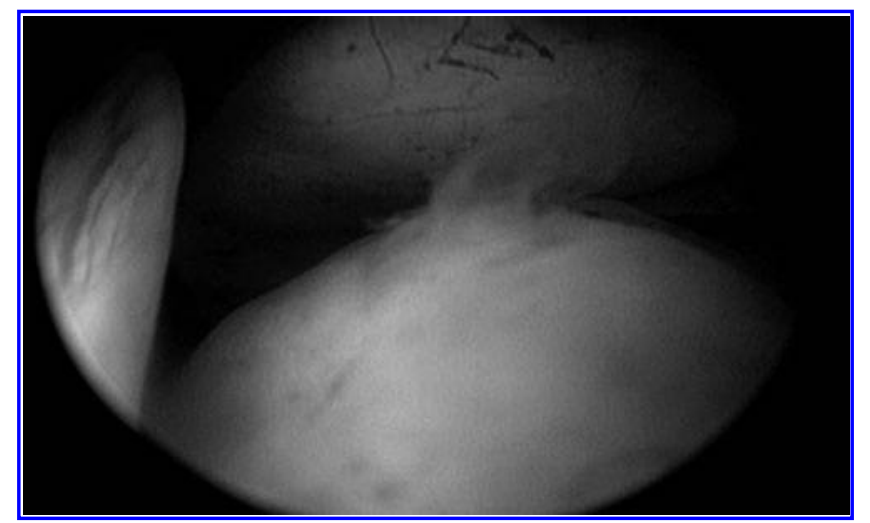

FIG. 3. Hysteroscopy picture illustrating some of the multiple submucous fibroids.

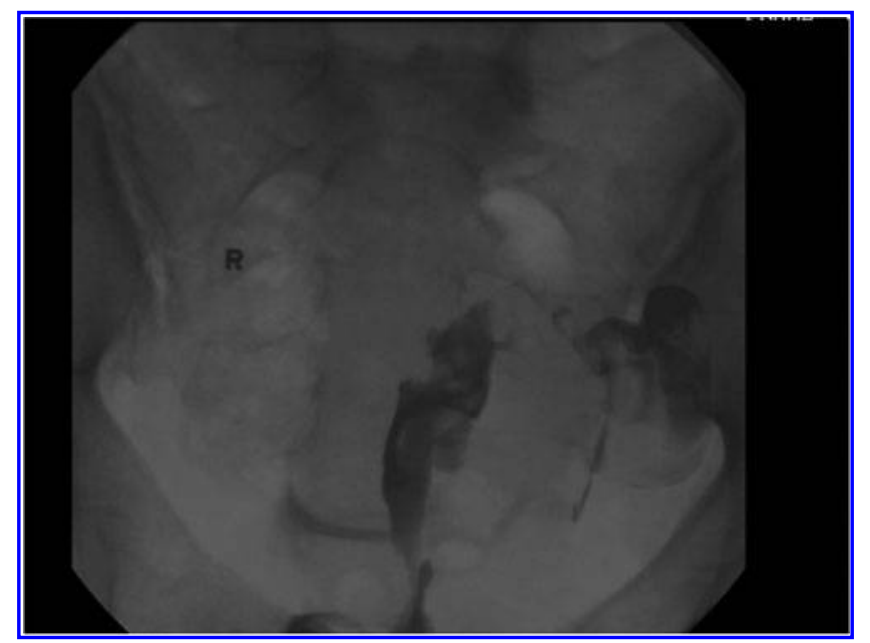

FIG. 4. Postoperative hysterosalpingogram demonstrated irregularity along the left uterine wall and an intrauterine scar that obliterated the right upper uterine segment.

conceiving in the future, and even hysterectomy should complications, especially uncontrolled hemorrhage, arise during the procedure. She was also made aware of the risk of uterine rupture and the need for a cesarean section should she conceive.
After 1 month of Luprorelin therapy, the abdominal myomectomy was performed. The pelvic cavity was explored, and there was no evidence of endometriosis. Her ovaries and fallopian tubes had a normal appearance. The uterus was bulky and firm, and no subserosal lesions were identified. The uterus was then injected with dilute pitressin, 5 units in $100 \mathrm{~mL}$ of saline, from the fundus to the lower uterine segment in the midline (anterior to posterior). The uterus was then bi-halved to expose the endometrial cavity; there were 15 fibroids that were either visualized or palpated and ranging in size from 0.5 to $1.5 \mathrm{~cm}$ (Fig. 5A, B). There was also a band of intrauterine adhesions identified in the right upper uterine segment, which was sharply dissected.

The myomas were then serially injected with pitressin to aid in adequate hemostasis. Serially incising the endometrium above each fibroid, dissecting off the endometrial lining with a hemostat, and bluntly resecting the fibroids were carefully performed (Fig. 6). Few figures of eight sutures were placed in the myometrium to obliterate the dead space where the intramural portion of these fibroids extended. Diluted indigo carmine was sprayed into the endometrial cavity to delineate the endometrial lining. Using magnifying glasses, care was taken to avoid suturing the endometrium. A $12 \mathrm{~F}$ Foley catheter was transcervically placed into the endometrial cavity and insufflated with $6 \mathrm{~mL}$ of saline to maintain endometrial distension to reduced scar formation (Fig. 7A). Attention was then turned to reconstructing the myometrium, a copious amount if the heparinized lactated ringer was used for irrigation. The primary closure of the myometrium was performed by placing a series of figures of eight sutures with 3-0 vicryl (Fig. 7B) in two layers. The serosa was closed with a baseball stitch of 3-0 vicryl sutures (Fig. 8). A hemovac was placed into the peritoneal, and the abdomen was closed in the usual fashion.

Postoperatively, the patient developed a temperature of $101 \mathrm{~F}$ and her hemoglobin dropped from 13.2 to 9.4. She was given intravenous antibiotics, and the hemovac was removed on postoperative day 3. She was discharged home on postoperative day 4. Her transcervical Foley was removed on postoperative day 9. She was placed on estrogen $1.25 \mathrm{mg}$ b.i.d. for 6 weeks followed by medroxyprogesterone $10 \mathrm{mg}$ for 10 days. Thereafter, an ultrasound was performed and
FIG. 5. (A, B) Pictures illustrating bihalved uterus exposing the endometrial cavity and the technique of removal of some of the submucous fibroids.

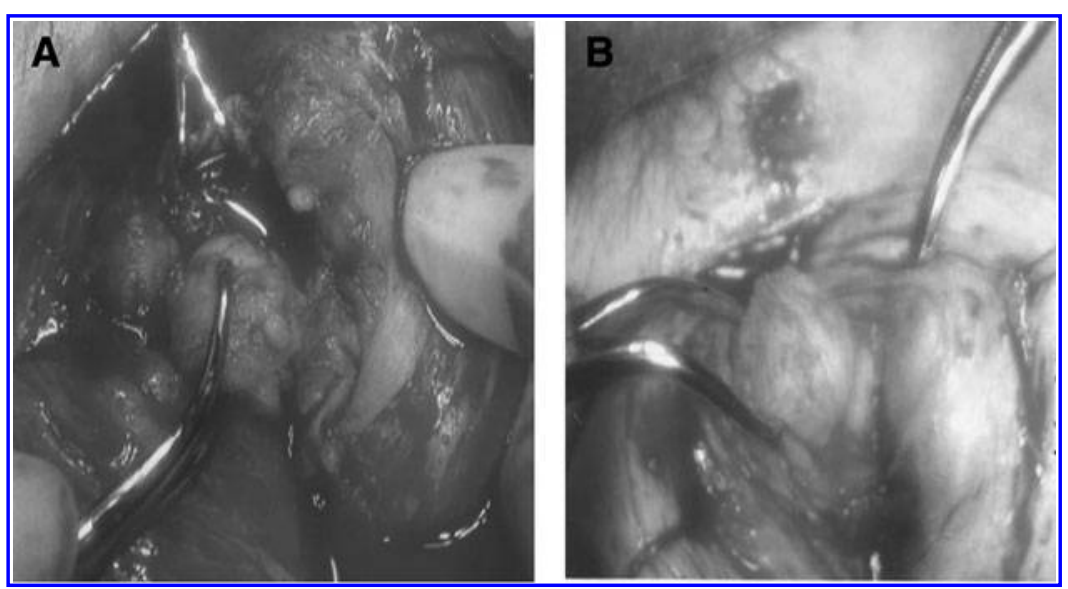




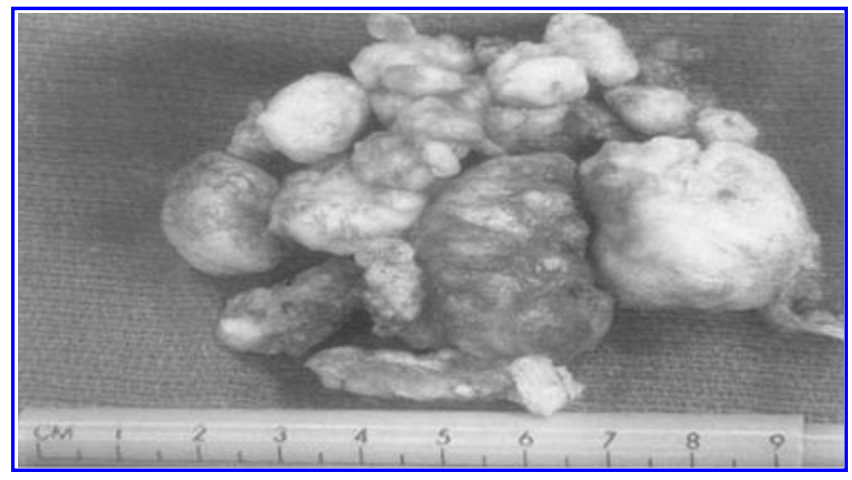

FIG. 6. Multiple fibroids after myomectomy ranging in size from 0.5 to $3 \mathrm{~cm}$.

there was no visible evidence of fibroids. A saline sonogram revealed minimal synechiae in the right upper uterine segment, but there was no evidence of submucous fibroids.

Twelve weeks postoperatively, the patient underwent in vitro fertilization (IVF) and embryo transfer. She was successfully impregnated after one cycle. She had an episode of vaginal bleeding during the first trimester. Her care was transferred to the perinatology service. She was given prophylactic steroids at 26 weeks of gestation. She presented to the hospital at 29 weeks of gestation with preterm premature rupture of membranes, with no signs of labor or infection. She was admitted to the antenatal unit and given a course of ampicillin and azithromycin. She had twice weekly biophysical profiles and nonstress testing during every shift. Ultrasonography was performed to monitor fetal growth. During her hospital course, her amniotic fluid volume remained normal despite her continued leakage of fluid. She experienced one episode of vaginal bleeding at 31 weeks of gestation. She underwent an amniocentesis, which ruled out infection and demonstrated fetal lung maturity. She was scheduled to have her cesarean section 2 weeks later.

Through a Pfannestiel incision, she had a primary low vertical cesarean section. She delivered a viable male infant weighing 6 pounds, 1 ounce with Apgar's of 6 at 1 minute and 9 at 5 minutes. Both fallopian tubes were evaluated and appeared normal. A small submucosal fibroid measuring
$<1.5 \mathrm{~cm}$ was noted and removed by simple blunt dissection. Her uterine incision was repaired with 0 vicryl in a running locked fashion. A second layer of this suture was used for hemostasis. The abdomen was closed in the usual fashion. Her postoperative course was uncomplicated.

\section{Discussion}

Uterine leiomyoma are estimated to account for $1 \%$ to $2 \%$ of infertility. ${ }^{2}$ The location of a fibroid and its size is the key factor regarding fertility. ${ }^{7}$ Several studies were done in the past to study the effect of the location of the fibroid on infertility: Elder-Geva et al. ${ }^{8}$ reported adverse effects on ongoing pregnancy rates in patients with submucosal and intrmural fibroids, but there was no observed difference in subserosal fibroids. Hart et al. ${ }^{9}$ studied the effect of small $(<5 \mathrm{~cm})$ intramural fibroids and found lower implantation and ongoing pregnancy rates in patients with fibroids compared with those with no fibroids. Pritts et al. ${ }^{7}$ showed that submucosal fibroids decrease fertility and myomectomy offers a benefit, and intramural fibroids decrease fertility but results of the therapy are unclear; however, subserous fibroids do not affect fertility and removal does not confer benefits.

Significantly lower implantation rates are associated with infertile women who have fibroids when compared with infertile controls with similar primary infertility diagnosis and no fibroids. ${ }^{3,6}$ A meta analysis done by Sunkara et al. showed a significant decrease in the live birth and clinical pregnancy rate in women with noncavity distorting intramural fibroids compared with those without fibroids, after IVF treatment. ${ }^{10}$ This was explained by Orisaka et al., who showed an abnormal uterine peristalsis pattern in women with fibroids during menstruation and mid-luteal phase (peri-implantation period) and that may affect embryo implantation. ${ }^{11}$

Our patient had multiple documented submucosal fibroids, so hysteroscopic resection was considered an appropriate initial approach. Unfortunately, due to the number, location, and depth of the fibroids, myomectomy was suboptimal and led to intrauterine scar tissue formation, the patient elected to undergo a second operation, and laparotomy with myomectomy was the decision this time. Garcia and Tureck observed a $47 \%$ pregnancy rate after abdominal

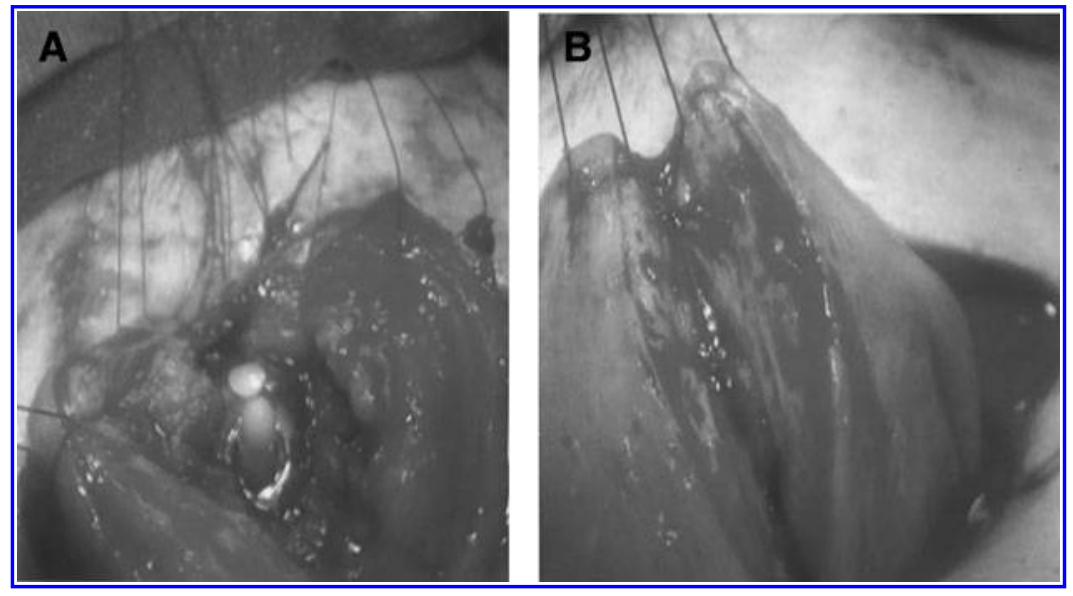

FIG. 7. (A, B) Pictures illustrating the reconstruction of the uterus with a transcervical Foley catheter placed in the endometrial cavity. 
FIG. 8. (A, B) Pictures of the uterus before and after closure of the serosal layer.

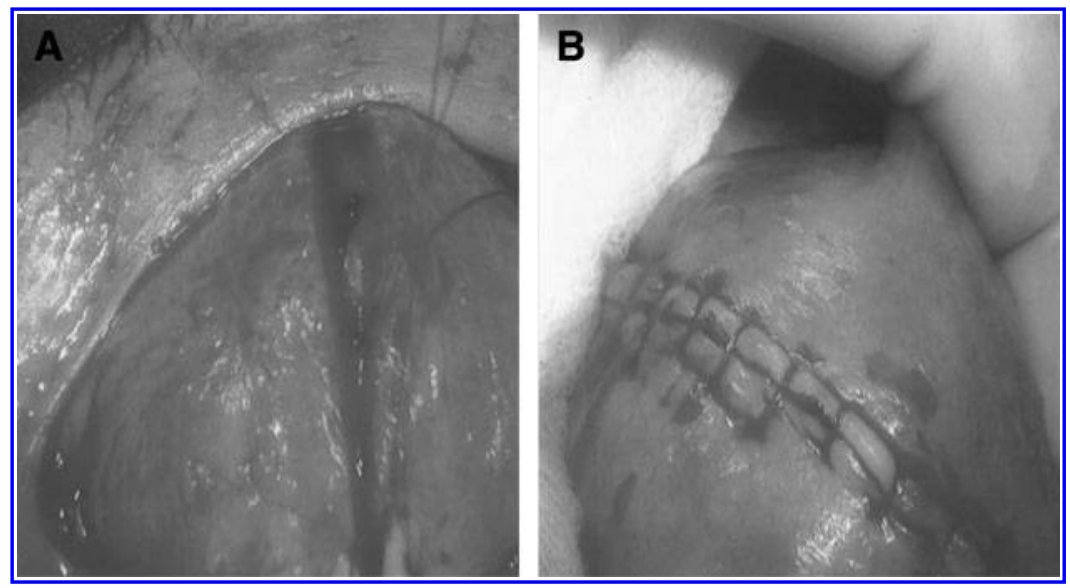

myomectomy of fibroids. ${ }^{12}$ Rosenfeld reported a $56.2 \%$ conception rate after abdominal myomectomy. ${ }^{13}$ Abdominal myomectomy was successfully done without compromising the patient's endometrial lining.

In our patient, workup revealed no additional etiology for her infertility. Multiple submucous myomas were the only identified etiology for her infertility. Our goal was to restore the uterine anatomy, treat the menorraghia, and enhance her conception rate. Initially, a partial hysteroscopic myomectomy was performed, in view of the number of submucous fibroids. Unfortunately, this resulted in partial intrauterine synechiae. Laparotomy with myomectomy seemed to be the next right approach for this patient. Initially, laparotomy with myomectomy was not done to avoid the complications that can result from abdominal myomectomy and its associated morbidity, mainly intrauterine and extrauteriune adhesions. In a study in which second-look laparotomy was performed after abdominal or laparoscopic myomectomy, adhesions were found in $36 \%$ of women. ${ }^{14}$ Other kinds of morbidity include blood transfusion, risk of uterine rupture with pregnancy due to uterine scars.

Myomectomy for the treatment of infertility remains debatable. This case is being reported to reinforce the concept that resection of multiple submucosal fibroids can have a positive impact on future fertility. Laparoscopy, laparotomy, and hysteroscopy are viable tools for surgical management. Each procedure has its drawbacks. This case demonstrated that the use of hysteroscopic myomectomy in the management of patients with multiple submucous fibroids and infertility can result in significant intrauterine synechiae that further compromise the patient fertility. The number, location, and difficulty in determining the extent of intramural involvement of the myoma may have contributed to the development of intrauterine scar tissue. During the abdominal myomectomy after bi-halving the uterus and the meticulous steps that were taken to maintain the integrity of the endometrial lining have helped in re-establishing a functional endometrial cavity.

\section{Conclusion}

Based on our experience with this case, we recommend the use of abdominal myomectomy after bi-halving the uterus for management of multiple sub-mucous fibroids from the outset. However, we would like to emphasize that such procedures should only be performed by an experienced reproductive surgeon who is familiar with procedures such as modified Tompkins metroplasty and Strassmann metroplasty.

\section{Acknowledgment}

The authors would like to thank Cheryl Anderson for preparation of this article.

\section{Disclosure Statement}

No competing financial interests exist.

\section{References}

1. Scott J, Gibbs R, Karlan B, Haney A. Danforths obstetric and gynecology, 9th ed. Philadelphia: Lippincott William and Wilkinis, 2003.

2. Buttram VC, Reiter RC. Uterine leimyomata: Etiology, symptomatology and management. Fertil Steril 1981;36: 433.

3. Pritts, EA. Fibroids and infertility: A systematic review of the evidence. Obstet Gynecol Surv 2001;56:484.

4. Deligdish L, Loewenthal M. Endometrial changes associated with myomata of uterus. J Clin Pathol 1970;23: 6776.

5. Richards PA, Richards PDG, Tiltman AJ. The ultrastructure of fibromyomatous myometrium and its relationship to infertility. Hum Reprod Update 1998;4:520.

6. Healy DL. Impact of uterine fibroids on ART outcome. Environ Health Perspect 2000;108:845.

7. Pritts EA, Parker WH, Olive DL. Fibroids and infertility: An updated systematic review of the evidence. Fertil Steril 2009;91:1215.

8. Elder-Geva T, Meagher S, Healy DL, Maclachlan V, Breheny S, Wood C. Effect of intramural, subserosal, and submucosal uterine fibroids on the outcome of assisted reproductive technology treatment. Fertil Steril 1998;70: 687.

9. Hart R, Khalaf Y, Yeong CT, Seed P, Taylor A, Braude P. A prospective controlled study of the effect of intramural uterine fibroids on the outcome of assisted conception. Hum Reprod 2001;16:2411. 
10. Sunkara SK, Khairy M, El-Toukhy T, Khalaf Y, Coomarasamy A. The effect of intramural fibroids without uterine cavity involvement on the outcome of IVF treatment: A systematic review and meta-analysis. Hum Reprod 2010; 25:418.

11. Orisaka M, Kurokawa T, Shukunami K, et al. A comparison of uterine peristalsis in women with normal uteri and uterine leiomyoma by cine magnetic resonance imaging. Eur J Obstet Gynecol Reprod Biol 2007;135:111.

12. Garcia CR, Tureck RW. Submucosal leiomyomas and infertility. Fertil Steril 1984;42:16.

13. Rosenfeld DL. Abdominal myomectomy for otherwise unexplained infertility. Fertil Steril 1986;46:328.
14. Dubuisson JB, Fauconnier A, Chapron C, Kreiker G, Nörgaard C. Second look after laparoscopic myomectomy. Hum Reprod 1998;13:2102.

Address correspondence to: Ahmed Abdelaziz, MD Division of Reproductive Endocrinology and Infertility Department of Obstetrics and Gynecology Hurley Medical Center 1 Hurley Plaza Flint, MI 48503

E-mail: aabdela1@hurleymc.com 
This article has been cited by:

1. Omar M. Abuzeid, John Hebert, Mohammad Ashraf, Mohamed Mitwally, Michael P. Diamond, Mostafa I. Abuzeid. 2017. Pediatric Foley Catheter Placement After Operative Hysteroscopy Does Not Cause Ascending Infection. Journal of Minimally Invasive Gynecology . [Crossref] 